Draft version September 23, 2018

Typeset using $\mathrm{LAT}_{\mathrm{E}} \mathrm{X}$ modern style in AASTeX61

\title{
BIMODAL FORMATION TIME DISTRIBUTION FOR INFALL DARK MATTER HALOS
}

\author{
Jinguing Shi, ${ }^{1,2,3}$ Huiyuan Wang, ${ }^{1,4}$ H.J. Mo,${ }^{5,6}$ Lizhi Xie,${ }^{7}$ Xiaoyu Wang,,${ }^{1,4}$ \\ Andrea Lapi, ${ }^{2,8,9}$ AND Ravi K. Sheth ${ }^{10}$
}

\footnotetext{
${ }^{1}$ Key Laboratory for Research in Galaxies and Cosmology, Department of Astronomy, University of Science and Technology of China, Hefei, Anhui 230026, China

${ }^{2}$ SISSA, Via Bonomea 265, I-34136 Trieste, Italy

${ }^{3}$ Kavli Institute for Astronomy and Astrophysics, Peking University, Beijing 100871, China

${ }^{4}$ School of Astronomy and Space Science, University of Science and Technology of China, Hefei 230026, China

${ }^{5}$ Department of Astronomy, University of Massachusetts, Amherst MA 01003-9305, USA

${ }^{6}$ Astronomy Department and Center for Astrophysics, Tsinghua University, Beijing 10084, China

${ }^{7}$ INAFAstronomical Observatory of Trieste, via G.B. Tiepolo 11, I-34143 Trieste, Italy

${ }^{8}$ INFN-Sezione di Trie ste, via Valerio 2, 34127 Trieste, Italy

${ }^{9}$ INAF-Osservatorio Astronomico di Trieste, via Tiepolo 11, 34131 Trieste, Italy

${ }^{10}$ Center for Particle Cosmology, University of Pennsylvania, 209 S. 33rd St., Philadelphia, PA 19104, USA
}

\begin{abstract}
We use a $200 h^{-1} \mathrm{Mpc}$ a side N-body simulation to study the mass accretion history (MAH) of dark matter halos to be accreted by larger halos, which we call infall halos. We define a quantity $a_{\mathrm{nf}} \equiv\left(1+z_{\mathrm{f}}\right) /\left(1+z_{\text {peak }}\right)$ to characterize the MAH of infall halos, where $z_{\text {peak }}$ and $z_{\mathrm{f}}$ are the accretion and formation redshifts, respectively. We find that, at given $z_{\text {peak }}$, their MAH is bimodal. Infall halos are dominated by a young population at high redshift and by an old population at low redshift. For the young population, the $a_{\mathrm{nf}}$ distribution is narrow and peaks at about 1.2, independent of $z_{\text {peak }}$, while for the old population, the peak position and width of the $a_{\mathrm{nf}}$ distribution both increases with decreasing $z_{\text {peak }}$ and are both larger than those of the young population. This bimodal distribution is found to be closely connected to the two phases in the MAHs of halos. While members of the young population are still in the fast accretion phase at $z_{\text {peak }}$, those of the old population have already entered the slow accretion phase at $z_{\text {peak }}$. This bimodal distribution is
\end{abstract}

Corresponding author: Jingjing Shi \& Huiyuan Wang

jingshi@mail.ustc.edu.cn; whywang@ustc.edu.cn 
not found for the whole halo population, nor is it seen in halo merger trees generated with the extended Press-Schechter formalism. The infall halo population at $z_{\text {peak }}$ are, on average, younger than the whole halo population of similar masses identified at the same redshift. We discuss the implications of our findings in connection to the bimodal color distribution of observed galaxies and to the link between central and satellite galaxies.

Keywords: dark matter - large-scale structure of the universe - galaxies:

halos - methods: statistical 


\section{INTRODUCTION}

In the standard cold dark matter paradigm, dark matter halos are the basic units of the large-scale structures of the Universe and the hosts within which galaxies form. A lot of effort has been devoted to understanding the assembly histories of individual halos, because these histories are expected to be linked directly to the properties of galaxies that form in halos (see Mo et al. 2010 for a review). Numerical simulations and analytical models have both demonstrated that dark matter halos grow hierarchically via the accretion and merger of smaller halos (Lacey \& Cole 1993; Springel et al. 2005). The mass accretion histories (MAHs) of dark matter halos are complex, and a number of formation times have been proposed to characterize the properties of the formation history of a halo (see e.g. Li et al. 2008). The distributions of these formation times are usually single-peaked (Lin et al. 2003), which is very different from the distribution of galaxies, which exhibits a bimodal distribution in star formation rate (SFR) and color (Blanton et al. 2003, 2005; Baldry et al. 2004; Wyder et al. 2007; Wetzel et al. 2012). Moreover, numerous studies have shown that the halo assembly history is correlated with many other halo properties, such as halo mass, halo structure, dynamical state (Lacey \& Cole 1993; Jing \& Suto 2002; Gao et al. 2004; Allgood et al. 2006; Hahn et al. 2007; Wang et al. 2011; Shi et al. 2015), as well as large scale environment (Sheth \& Tormen 2004; Gao et al. 2005; Wang et al. 2007; Gao \& White 2007; Jing et al. 2007).

So far the investigations have focussed on the main trunk of the halo merger trees that reflect the evolution history of the main progenitors of a halo. To fully understand the halo assembly, halos on the subbranches of the merger trees should also been taken into account. Whereas the main trunk is related to the formation of the central galaxy in a halo, the sub-branches are related to the formation of satellite galaxies. The evolutionary history of subbranch halos can be divided into two phases: one before a halo has merged into a bigger halo and the other after the halo has merged to become a subhalo. In the first phase, the halos (hereafter referred to as infall halos) accrete material similarly to the main trunk halos, as they themselves are independent halos. They become substructures of larger halos, usually referred to as subhalos, only after the accretion. During the subhalo phase, the subbranch halos evolve in various ways due to interactions with the host halo, such as dynamical friction (Chandrasekhar 1943; Oguri \& Lee 2004; Hashimoto et al. 2003; Jiang et al. 2008), tidal heating and stripping (Hayashi et al. 2003; Taylor \& Babul 2004; Gan et al. 2010; Han et al. 2016), back-splashing (Ludlow et al. 2009), and impulsive encounters (van den Bosch 2017). There have been many studies of subhalo properties (Tormen 1997; Vitvitska et al. 2002; Gao et al. 2004; Benson 2005; Wang et al. 2005; Giocoli et al. 2008; Wetzel 2011; Jiang et al. 2015; Shi et al. 2015; Xie \& Gao 2015). In contrast, the mass assembly histories of the infall population, have so far drawn only little attention; the only related work known to us is Sheth (2003) where a simple Poisson model is developed to investigate the formation time distribution of infall halos. 
As mentioned above, infall halos are the hosts in which satellite galaxies we observed today form and evolve. Once these galaxies become satellite galaxies, they are expected to experience satellite-specific processes, such as tidal and ram pressure stripping (Gunn \& Gott 1972; Abadi et al. 1999; Quilis et al. 2000). To quantify the efficiency of these processes, one usually compares these satellites with central galaxies of similar stellar mass (van den Bosch et al. 2008; Weinmann et al. 2009; Pasquali et al. 2010; Wetzel et al. 2012; Peng et al. 2012; Knobel et al. 2013; Bluck et al. 2014; Fossati et al. 2017). This is only valid under the assumption that the halo assembly histories of infall halos, which are the progenitors of the subhalos hosting satellite galaxies, are similar to the assembly histories of the halos hosting central galaxies. Clearly, this assumption needs to be checked by comparing the formation histories between the two kinds of halos.

There is growing evidence that the star formation in a galaxy is correlated with the assembly history of its host halo. For example, Bray et al. (2016) found that galaxy color is correlated with halo formation time in the cosmological hydrodynamic simulation "Illustris" (e.g. Vogelsberger et al. 2014), in the sense that redder galaxies tend to live in older halos. Wang et al. (2017) found that the quenching probability, defined as the probability for a galaxy to be quenched, is related to the formation time of the host halo identified in constrained simulations. In particular, the subhalo abundance matching model, which links the formation time of the host halo to galaxy color (Hearin \& Watson 2013; Hearin et al. 2014; Chaves-Montero et al. 2016; Paranjape et al. 2015), can reproduce the observed clustering and cosmic shear signals separately for red and blue galaxies, as well as the observed "galactic conformity" (Hearin et al. 2014; Watson et al. 2015; Hearin et al. 2016). All these suggest that it is important to study the assembly histories of infall in order to understand the evolution of satellite galaxies.

In this paper, we present a detailed analysis of the mass accretion histories of infall halos. In Section 2, we describe the numerical simulation we use, the construction of halo merger trees, and the method to identify infall halos. In Section 3, we present our main results of the formation time distribution for infall halos, including the finding of bimodality in the distribution. In Section 4, we try to explain the bimodality using the fact that halos have two distinct accretion phases, and compare the properties

of infall halo population with the general halo population. Finally, in Section 6 we summarize our main results and discuss their implications for galaxy formation.

\section{NUMERICAL SIMULATION AND DARK MATTER HALOS}

\subsection{The Simulation and Halo Merger Trees}

The N-body simulation used here was carried out with Gadget-2 (Springel 2005), adopting a flat $\Lambda \mathrm{CDM}$ cosmology with parameters consistent with WMAP9 data (Hinshaw et al. 2013): $\Omega_{\Lambda, 0}=0.718, \Omega_{\mathrm{m}, 0}=0.282, \Omega_{\mathrm{b}, 0}=0.046, h=$ $H_{0} /\left(100 \mathrm{~km} \mathrm{~s}^{-1} \mathrm{Mpc}\right)=0.697, \sigma_{8}=0.817$, and $n_{s}=0.96$. The CDM density field is 
traced by $2048^{3}$ particles, each with a mass of $m_{\mathrm{p}} \approx 7.29 \times 10^{7} h^{-1} \mathrm{M}_{\odot}$, in a cubic box of $200 h^{-1} \mathrm{Mpc}$ in a side. The gravitational force is softened isotropically on a co-moving length scale of $2 h^{-1} \mathrm{kpc}$ (Plummer equivalent). Outputs are made at 100 snapshots from $z=20$ to $z=0$ equally spaced in the logarithm of the expansion factor.

Dark matter halos are first identified using the FOF algorithm (Davis et al. 1985) with a linking length of $0.2 b$, where $b$ is the mean inter particles separation, and all halos with at least 20 particles are selected. We then use the SUBFIND algorithm (Springel et al. 2001) to identify gravitationally bound substructures within each FOF halo. The most massive substructure in a FOF halo is called the main halo, while all the other substructures are referred to as subhalos. The virial mass $\left(M_{\mathrm{vir}}\right)$ of a main halo is defined as the mass contained in a spherical volume, centered on the minimum of the gravitational potential well, within which the average density is $200 \rho_{\text {crit }}$, with $\rho_{\text {crit }}$ being the critical density of the universe. We do not measure the current masses of subhalos, as they are usually strongly stripped; rather, we measure their masses before they are incorporated into their hosts (see below).

The construction of halo merger trees is based on the SUBFIND catalogs using the algorithm described in Springel et al. (2005) (see Boylan-Kolchin et al. 2009 for a more detailed description). Briefly, the member particles of a gravitationally bound substructure are assigned a weight that decreases with the binding energy calculated using all the particles in it. A search is then made in the subsequent snapshot for substructures (including both main halos and subhalos) that contain some of the particles of the substructure in question. The one which contains the largest weighted number of particles of the substructure is chosen as the descendent of the substructure in the snapshot. This method allows one to accurately trace the complex history of a substructure even in the cases where a main halo becomes a subhalo or where a subhalo is ejected so as to become a main halo again. For a FOF halo at redshift zero, the merger tree usually contains many branches, including one main trunk and many sub-branches, with the former tracing the main progenitors of the main halo in the FOF halo back in time, where the main progenitor is defined to be the most massive progenitor in the previous snapshot. The other parts of the merger tree are referred to as subbranches. Note that the branch that ends up in a subhalo at redshift zero is also referred to as a subbranch.

\subsection{Infall Halos, Accretion Time and Formation Time}

We define an infall halo to be one that is about to merge with a bigger halo. More specifically, infall halos are main halos on the sub-branches of merger trees. They eventually either become subhalos of larger FOF halos (the survived subhalos) or get totally disrupted at $z=0$. We further require that the descendant an infall halo has crossed the virial radius of the main trunk halo at least once. This selection criterion can effectively remove halos that are temporarily linked to the main trunk halos (see 
e.g. Tinker et al. 2008), thus treated as sub-branch halos, due to numerical effects. However, it will also remove some true infall halos. Our tests show that the bimodal distribution and our conclusions won't change significantly if we discard this criterion.

An infall halo can be accreted to become the subhalo of a larger halo either on a sub-branch or on the main trunk (Lacey \& Cole 1993; Springel et al. 2005). It is referred to as a first-order infall halo in the former case, and a higher-order infall halo in the latter. Since higher-order infall halos may become subhalos in a subhalo and may contribute significantly to the substructures in the final FOF halos, they are also included in our analysis. Moreover, some halos may have been accreted at an earlier time by larger halos, but subsequently ejected and become independent main halos at $z=0$ (Lin et al. 2003; Gill et al. 2005; Wang et al. 2009; Ludlow et al. 2009; Li et al. 2013). These halos, called "backsplash halos", are excluded from our infall halo samples. However, these halos are treated as main trunk halos and their own infall halos are included in our analysis. We will also include another population of infall halos, called "wavering" infall halos, which entered their hosts at high $z$, but left and re-entered the hosts later.

Once an infall halo is identified, we trace its merger tree and find the redshift at which its $M_{\text {vir }}$ reaches the peak value in its lifetime $\left(M_{\text {peak }}\right)$. This redshift is denoted $z_{\text {peak }}$ in the following. In our analysis, we ignore the part of the history after the infall halo is accreted to become a subhalo. Since a halo in general loses mass in the subhalo phase, ignoring this part of the history has no significant impact on the measurements of $z_{\text {peak }}$ and $M_{\text {peak }}$. By construction, all infall halos at $z_{\text {peak }}$ are themselves main halos. Following Xie \& Gao (2015), we refer to $z_{\text {peak }}$ as the accretion time of the infall halo. Other definitions of the accretion time include the time when the merger of the infall halo with its host occurs, i.e. the time when an infall halo first becomes a subhalo (see Li \& Mo 2009; Giocoli et al. 2008), and the time at which the maximum circular velocity $\left(V_{\max }\right)$ of a halo reaches the maximum value in its lifetime (Conroy et al. 2006; Nagai \& Kravtsov 2005). We will denote the corresponding redshifts by $z_{\text {inf }}$ and $z_{\mathrm{vp}}$, respectively. We will discuss how our results change with the definitions of accretion time in Section 3.4.

For each infall halo, we estimate a formation time, corresponding to a redshift $z_{\mathrm{f}}$, defined as the time at which the halo reaches half of the halo mass at accretion time for the first time. In most part of this paper, we adopt $z_{\text {peak }}$ to define accretion time, so the halo mass at $z_{\mathrm{f}}$ equals to $M_{\text {peak }} / 2$. Nevertheless, in Section 3.4, we adopt two other definitions for the accretion time, $z_{\text {inf }}$ and $z_{\mathrm{vp}}$, so that the corresponding formation times are also different. Thus, for each infall halo, we are able to obtain two characteristic times: the accretion time (which is also called the infall time) and the formation time. By definition, the formation time is always larger than the accretion time.

\subsection{Halo Selection}



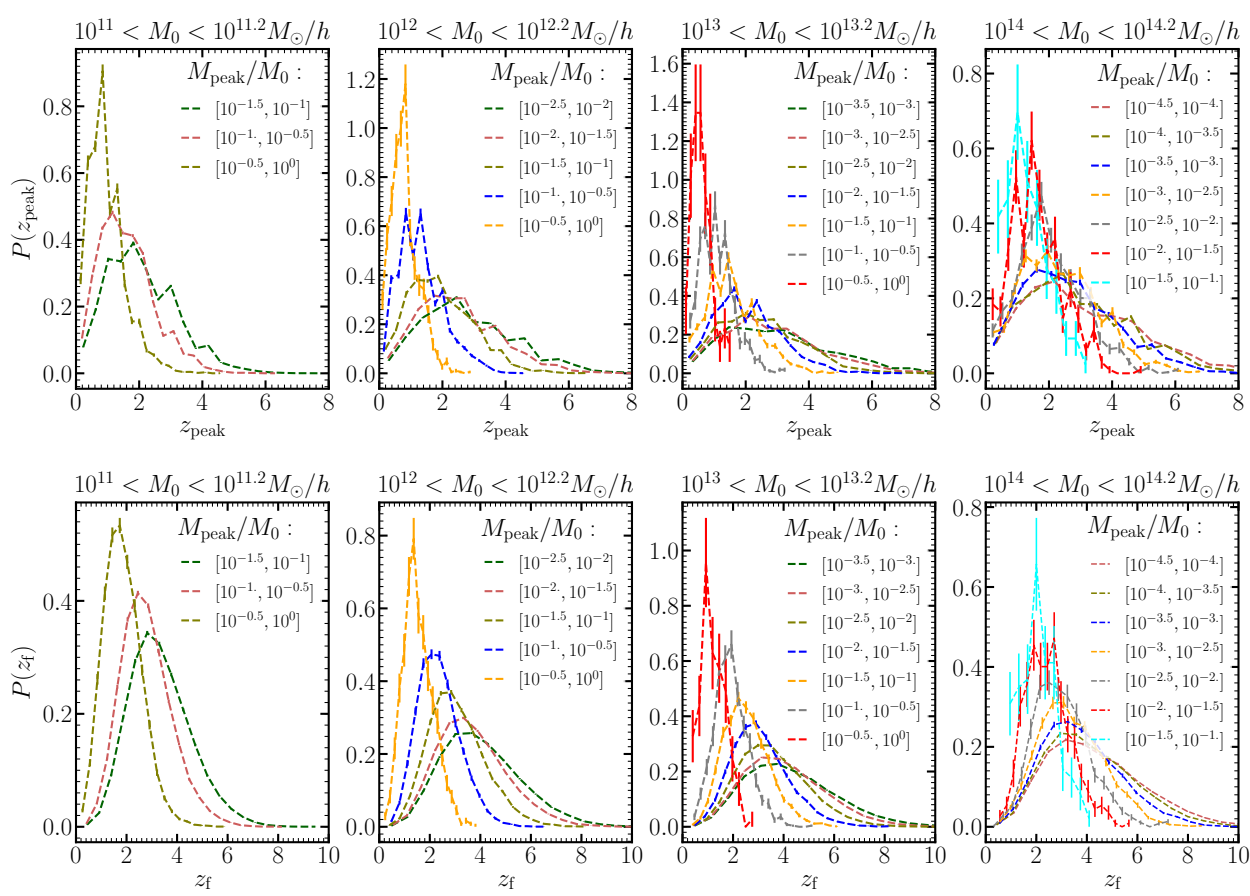

Figure 1. Upper panel: the distribution of the accretion redshfit $z_{\text {peak }}$ for infall halos of various $M_{0}$ and $M_{\text {peak }}$. Lower panel: the same as the upper panel, but for the formation redshift $z_{\mathrm{f}}$ of infall halos. Error bars show Poisson errors.

In order to obtain $z_{\mathrm{f}}$ reliably, we only consider infall halos with $M_{\text {peak }}>100 m_{\mathrm{p}}$. Throughout the paper, we use $M_{0}$ to denote the mass of the main trunk halo at $z=0$, and restrict our analysis to halos with $10^{11}<M_{0}<6 \times 10^{14} h^{-1} \mathrm{M}_{\odot}$, which contain at least 1,300 particles. With these selections, our final sample contains 191,166 main trunk halos at $z=0$ and 2,402,610 infall halos. We sometimes also use 'host halo' to refer to the halo into which an infall halo is accreted. It should emphasized, however, that host halos are not necessarily main trunk halos, since the main halos on subbranches can also accrete halos and thus be called host halos. Figure 1 shows separately the probability distributions of $z_{\text {peak }}$ and $z_{\mathrm{f}}$ for four $M_{0}$ bins (as shown in each column) and several $M_{\text {peak }}$ bins (as indicated by the labels in each panel). Both quantities have single-peaked (unimodal) distributions. In general, more massive infall halos tend to have lower $z_{\text {peak }}$ and lower $z_{\mathrm{f}}$.

\section{BIMODAL FORMATION TIME DISTRIBUTION}

\subsection{Bimodal distributions of formation times for infall halos}

We show the formation redshift distributions in the left panel of Figure 2 for infall halos with $M_{\text {peak }}>100 m_{\mathrm{p}}$ and $10^{11}<M_{0}<6 \times 10^{14} h^{-1} \mathrm{M}_{\odot}$ at various $z_{\text {peak }}$. To compare results for different $z_{\text {peak }}$ and to better understand the results, we actually show the distributions of

$$
a_{\mathrm{nf}} \equiv \frac{1+z_{\mathrm{f}}}{1+z_{\text {peak }}} .
$$



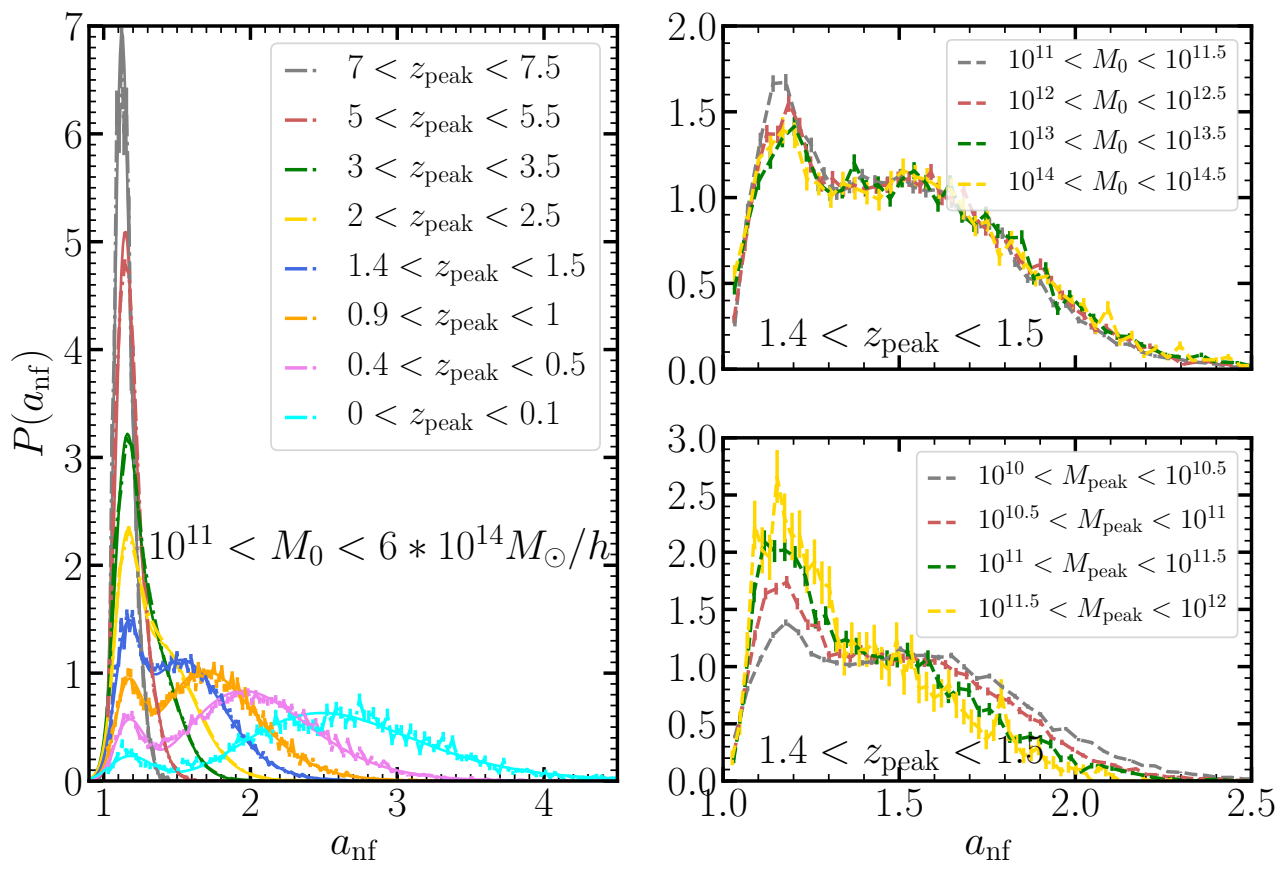

Figure 2. Left panel: The distributions of $a_{\mathrm{nf}} \equiv\left(1+z_{\mathrm{f}}\right) /\left(1+z_{\text {peak }}\right)$ for infall halos with $M_{\text {peak }}>100 m_{\mathrm{p}}$ at various $z_{\text {peak }}$. Right Panel: the $a_{\mathrm{nf}}$ distributions for halos of various $M_{0}$ (upper panel), and various $M_{\text {peak }}$ (lower panel). In both panels, results are shown only for $1.4<z_{\text {peak }}<1.5$. Solid lines are double log-normal fitting curves. Error bars show Poisson errors.

$a_{\mathrm{nf}}$ can be used to describe the relative age of infall halos that are accreted at the same accretion time. Interestingly, the $a_{\text {nf }}$ distribution is bimodal, while the formation time distributions shown in Figure 1 are clearly unimodal. This indicates that the bimodality appears only for fixed $z_{\text {peak }}$.

For halos with $z_{\text {peak }}>5$, the distributions of $a_{\text {nf }}$ are narrow and peak at $a_{\text {nf }} \sim 1.2$. This implies a uniform accretion pattern, as we will discuss below. As $z_{\text {peak }}$ decreases, a second peak appears in the distribution and becomes increasingly more important. At $z_{\text {peak }} \sim 2$, the two components become comparable in height. As $z_{\text {peak }}$ approaches zero, the peak at low $a_{\mathrm{nf}}$ almost disappears. For convenience, we refer halos in the low $a_{\mathrm{nf}}$ mode as the young population, and those in the high $a_{\mathrm{nf}}$ mode as the old population.

To describe the redshift evolution of the two populations, we fit the $a_{\mathrm{nf}}$ distributions with a double log-normal function:

$$
P\left(a_{\mathrm{nf}} \mid z_{\text {peak }}\right)=\omega \mathcal{N}_{\log }\left(\mu_{1}, \sigma_{1}^{2}\right)+(1-\omega) \mathcal{N}_{\log }\left(\mu_{2}, \sigma_{2}^{2}\right)
$$

where $\mu_{1}<\mu_{2}$ and $\mathcal{N}_{\log }\left(\mu, \sigma^{2}\right)$ represents the log-normal function. The fitting is performed with the non-linear least square method. As shown in Figure 2, the distributions can be well fitted by the double log-normal model. The two populations 
clearly evolve differently. Our best fitting result suggests that, for the young population, the peak position and width of the distribution change little with $z_{\text {peak }}$, with the peak staying at $a_{\mathrm{nf}} \sim 1.2$. In contrast, both the peak position and dispersion decrease significantly with increasing $z_{\text {peak }}$ for the old population.

\subsection{Dependence on $M_{\text {peak }}, M_{0}$, and sub-classes of infall halos}

For the main trunk halos, it is known that the formation time depends on halo mass. We thus check whether the bimodality is related to halo mass. Since the bimodal feature is the most prominent for $1<z_{\text {peak }}<2$, we show the results for infall halos with $1.4<z_{\text {peak }}<1.5$. The upper and lower right panels of Figure 2 show the $\left(1+z_{\mathrm{f}}\right) /\left(1+z_{\text {peak }}\right)$ distributions for various $M_{0}$ and $M_{\text {peak }}$, respectively. The bimodality is clearly present in all the mass bins shown. The dependence on $M_{0}$ is rather weak; there is a very weak trend for the fraction of the young population to decrease with increasing $M_{0}$. The dependence on $M_{\text {peak }}$ is stronger, with the young population fraction increasing significantly with $M_{\text {peak }}$. Similar to the result for the total sample shown in the left panel of Figure 2, there is no significant change in the peak position and width for the young population. Both the peak and width of the distribution increase with decreasing $M_{\text {peak }}$ for the old population, which is similar to the main trunk halos (e.g. Wang et al. 2007).

An interesting question is whether the existence of the bimodality depends on the final states of the infall halos. In the left panel of Figure 3, we show the results for infall halos that survive as subhalos at redshift zero. Since only few infall halos with $z_{\text {peak }}>4$ can survive in their final hosts, the results for the two highest redshift bins are not shown. As one can see, the bimodality in the distribution of $a_{\mathrm{nf}}$ is very similar to that for the total population, indicating that the bimodal feature is independent of the final states of the infall halos.

Next, we examine first-order infall halos, namely halos that fall directly into the main trunk halos, no matter whether they survive or are fully disrupted (see figure 1 of Jiang \& van den Bosch (2014) for a depiction of such halos). The results are shown in the right panel of Figure 3. Again, the difference from the total population is quite small. We have also checked other two sub-populations: the "wavering" population, which entered their host at high redshift, but then left and re-entered the hosts later; infall halos that are accreted by the 'backsplash' halos. These two sub-populations also exhibit clear bimodality in their $a_{\mathrm{nf}}$ distributions.

\subsection{Resolution tests}

To examine whether or not the observed bimodality can be significantly biased or even caused by the mass resolution of the simulation, we have made the same analysis using a simulation in a $500 \mathrm{~h}^{-1} \mathrm{Mpc}$ box run with $3072^{3}$ particles (Li et al. 2016). This simulation uses the same cosmology as the simulation used above, but has a mass resolution that is lower by a factor of 4.63. For the same ranges of $M_{0}, M_{\text {peak }}$, and $z_{\text {peak }}$, the $a_{\mathrm{nf}}$ distributions obtained from the two simulations are indistinguishable. 


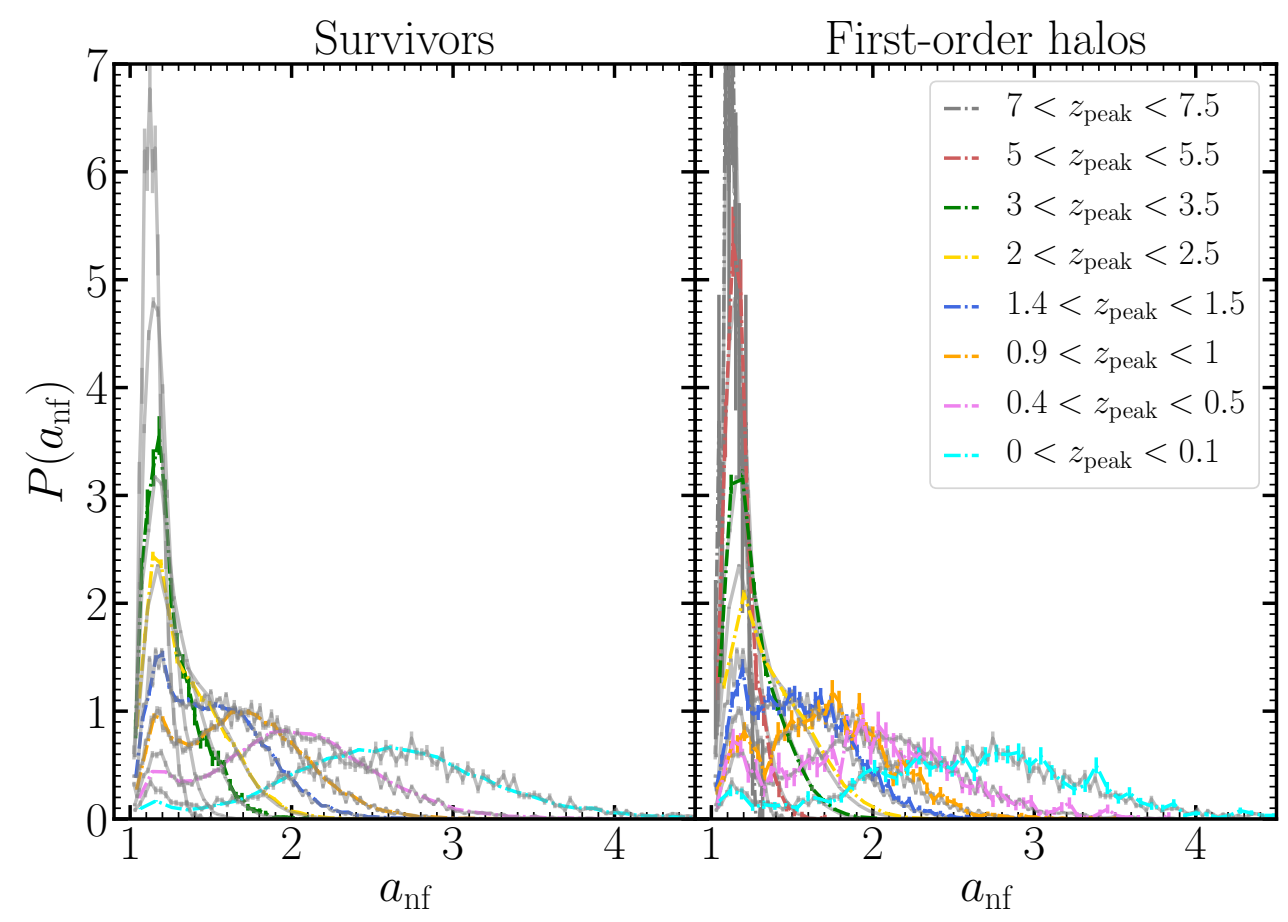

Figure 3. Left panel shows the distributions of $a_{\mathrm{nf}}$ for infall halos that survive as subhalos at $z=0$. Right panel shows results for halos that infall directly onto the main trunk. The solid gray lines are the results for the whole population as shown in the left panel of figure 2. The error bars show Poisson errors.

We have also applied our analysis to two suites of high-resolution simulations of individual dark matter halos from the Phoenix and the Aquarius projects (Springel et al. 2008; Gao et al. 2012). These have mass resolutions that are higher than our simulation by a factor of more than 10 and 1000, respectively. Similar bimodal distributions are also found for these individual halos. This demonstrates that our results are not affected by the resolution of our simulation.

\subsection{Dependence on the definition of accretion times}

We have also checked how our results may vary with different definitions for the accretion time. In addition to $z_{\text {peak }}$, there are two other commonly used definitions: the redshift, $z_{\mathrm{vp}}$, at which the maximum circular velocity $\left(V_{\max }\right)$ of a halo reaches the maximum value in its lifetime (Conroy et al. 2006; Nagai \& Kravtsov 2005); and the redshift $z_{\text {inf }}$ at which a halo becomes a subhalo for the first time (Li \& Mo 2009; Shi et al. 2015). Similarly, we define the formation redshift as the redshift at which the infall halo first reaches half of its mass at the accretion redshift for these two definitions of accretion time. We find that using $z_{\mathrm{vp}}$ instead of $z_{\text {peak }}$ does not change the bimodal distribution of the formation redshift, but the bimodality becomes less prominent when $z_{\text {inf }}$ is used instead of $z_{\text {peak }}$. About $50 \%$ of the infall halos have $z_{\text {inf }}=z_{\text {peak }}$, and so the reduced prominence comes from the other infall 
halos. Among them, about $60 \%$ have $z_{\text {inf }}<z_{\text {peak }}$, and these halos are expected to have experienced tidal stripping before falling into their hosts. For the $40 \%$ that have $z_{\text {inf }}>z_{\text {peak }}$, their masses actually grow after being accreted by larger halos. There are two possibilities for this after-accretion growth. First, the halos may be ejected from their hosts and grow mass after $z_{\text {inf }}$; second, the halos are not accreted at $z_{\text {inf }}$, but linked with their 'hosts' by a temporary bridge (see e.g. Tinker et al. 2008). Because of these uncertainties, we believe that $z_{\text {peak }}$ is a better choice than $z_{\text {inf }}$ for defining the accretion of a halo.

\section{FAST AND SLOW ACCRETION}

The formation time, $z_{\mathrm{f}}$, is only one of many parameters that characterize the mass assembly history (MAH) of a halo. To obtain more insight about halo growth, we show in Figure 4 the MAH for infall halos randomly selected from the two populations. For each halo selected, we plot $M_{\mathrm{vir}}(z)$ (normalized by $\left.M\left(z_{\text {peak }}\right)\right)$ as a function of $(1+$ $z) /\left(1+z_{\text {peak }}\right)$, where $M_{\text {vir }}(z)$ is mass of the most massive progenitor at $z$. Motivated by the presence of the bimodal feature, we use infall halos with $a_{\mathrm{nf}}<1.3$ to represent the young population and the ones with $a_{\mathrm{nf}}>1.5$ to represent the old population. Clearly, the two populations have quite different MAH. The old population is characterized by a fast mass growth at high redshift, and the mass accretion rate slows down before reaching $M_{\text {peak }}$. In contrast, the young population shows a much faster growth than the old one, in particularly at low $a_{\mathrm{nf}}$. These demonstrate clearly that the presence of the bimodal distribution in $a_{\mathrm{nf}}$ is closely related to the MAH of halos.

The mass accretion history of infall halos plays a key role in our understanding of the formation and evolution of satellite galaxies. It is thus important to investigate the origin of the bimodal distribution. Zhao et al. (2003a,b) found that the MAH of a halo can be divided into a fast accretion phase and a slow accretion phase, and the average $\mathrm{MAH}$ can be described in the form,

$$
\frac{M_{\mathrm{vir}}(z)}{M_{\mathrm{vir}}\left(z_{\mathrm{tp}}\right)}=\frac{t^{0.3}}{1-b+b t^{-1.8 b}}
$$

where $t \equiv \rho_{\text {vir }}\left(z_{\mathrm{tp}}\right) / \rho_{\text {vir }}(z)$ and $z_{\mathrm{tp}}$ stands for the transition redshift between the two phases. The parameter $b$ is 0.75 for the fast accretion phase $\left(z>z_{\mathrm{tp}}\right)$ and 0.42 for the slow accretion phase $\left(z<z_{\mathrm{tp}}\right)$. Since we assume $\rho_{\mathrm{vir}}(z)=200 \rho_{\text {crit }}(z)$, the dimensionless time variable can be written as $t=\left[H^{2}\left(z_{\mathrm{tp}}\right) / H^{2}(z)\right]$. This formula suggests that, at high redshift, most halos are in the fast accretion phase, but at redshift zero, most halos are in the slow accretion phase.

To understand the connection of the bimodal distribution to the two-phase accretion, we consider two extreme cases. First, suppose that an infall halo has $z_{\text {peak }} \gg z_{\text {tp }}$, and so $z_{\mathrm{f}} \gg z_{\text {tp }}$. We then have $t_{\mathrm{f}}=H^{2}\left(z_{\mathrm{tp}}\right) / H^{2}\left(z_{\mathrm{f}}\right) \ll 1$, and

$t_{\text {peak }}=H^{2}\left(z_{\mathrm{tp}}\right) / H^{2}\left(z_{\text {peak }}\right) \ll 1$. Since this halo stays in the fast accretion phase before being accreted at $z>z_{\text {peak }}$, we have $b=0.75$ and $M_{\text {vir }}(z) / M_{\text {vir }}\left(z_{\text {tp }}\right) \simeq t^{1.65} / 0.75$. 


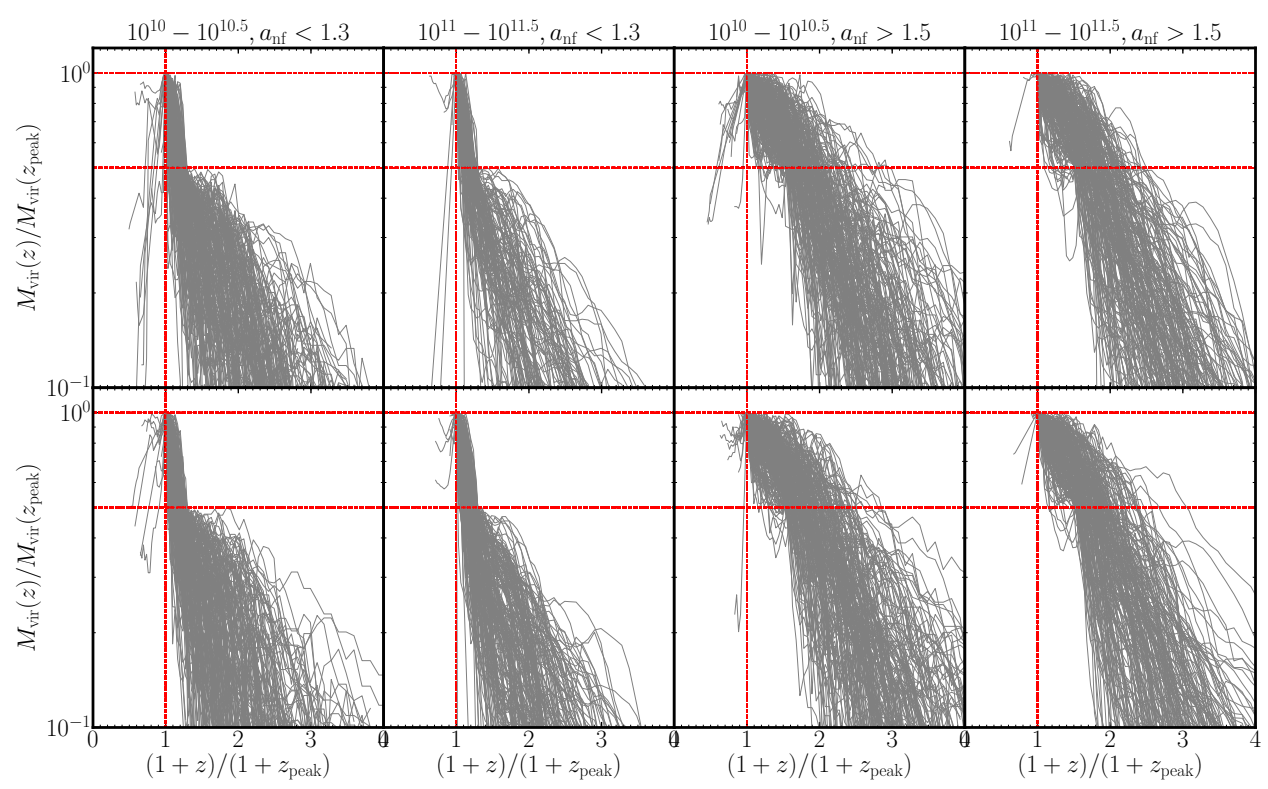

Figure 4. Mass accretion histories for young (left two columns) and old (right two columns) infall halos with different $M_{\text {peak }}$ and $M_{0}$. Here the young population has halos with $a_{\mathrm{nf}}<$ 1.3 while the old halo population has $a_{\mathrm{nf}}>1.5$. The upper panels show the results for $10^{12}<M_{0}<10^{13} h^{-1} \mathrm{M}_{\odot}$; the lower panels for $10^{13}<M_{0}<10^{14} h^{-1} \mathrm{M}_{\odot}$. Two mass ranges for infall halos are chosen: $10^{10}-10^{10.5} h^{-1} \mathrm{M}_{\odot}$ and $10^{11}-10^{11.5} h^{-1} \mathrm{M}_{\odot}$, as indicated in each panel. The upper and lower horizontal red lines indicate $M(z)=M_{0}$ and $M(z)=M_{0} / 2$, respectively.

We can define a new quantity,

$$
\log x \equiv \log \left[H^{2}\left(z_{\mathrm{f}}\right) / H^{2}\left(z_{\text {peak }}\right)\right]=\log \left[t_{\text {peak }} / t_{\mathrm{f}}\right]
$$

For this halo, this equation can be rewritten as $\log x \simeq \log \left[M_{\text {vir }}\left(z_{\text {peak }}\right) / M_{\text {vir }}\left(z_{\mathrm{f}}\right)\right] / 1.65$. By definition, $M_{\text {vir }}\left(z_{\mathrm{f}}\right)=M_{\text {vir }}\left(z_{\text {peak }}\right) / 2=M_{\text {peak }} / 2$, so we have $\log x \simeq 0.18$. In the second case, where the infall halo is assumed to have $z_{\text {peak }} \ll z_{\text {tp }}$ and $z_{\mathrm{f}} \ll z_{\text {tp }}$, we have $t_{\mathrm{f}} \gg 1$ and $t_{\text {peak }} \gg 1$, and so the halo is in the slow accretion phase $(b=0.42)$. Its MAH can then be simplified as $M_{\mathrm{vir}}(z) / M_{\mathrm{vir}}\left(z_{\mathrm{tp}}\right) \simeq t^{0.3} / 0.58$ and we have $\log x \simeq 1$. For each infall halo, we have its $z_{\mathrm{f}}$ and $z_{\text {peak }}$, and so can derive its $\log x=\log \left[H^{2}\left(z_{\mathrm{f}}\right) / H^{2}\left(z_{\text {peak }}\right)\right]$. Figure 5 shows the distributions of $\log x=$ $\log \left[H^{2}\left(z_{\mathrm{f}}\right) / H^{2}\left(z_{\text {peak }}\right)\right]$ for halos with $M_{\text {peak }}>100 m_{\mathrm{p}}, 10^{11}<M_{0}<6 \times 10^{14} h^{-1} \mathrm{M}_{\odot}$, and various $z_{\text {peak }}$. The colors are coded in the same way as in the left panel in Figure 2. The new quantity also shows a clear bimodal distribution. In particular, the distributions for the young population roughly peak around $\log x=0.18$ for most values of $z_{\text {peak }}$. This suggests strongly that the young population halos are in the fast accretion phase and are far from the turning point (i.e. $z_{\mathrm{f}}>z_{\text {peak }} \gg z_{\mathrm{tp}}$ ). The situation for the old population looks more complicated. The peak of the distribution increases with decreasing $z_{\text {peak }}$ and ranges from $\sim 0.3$ to $\sim 1$. Only for $z_{\text {peak }} \sim 0$ is the peak of 


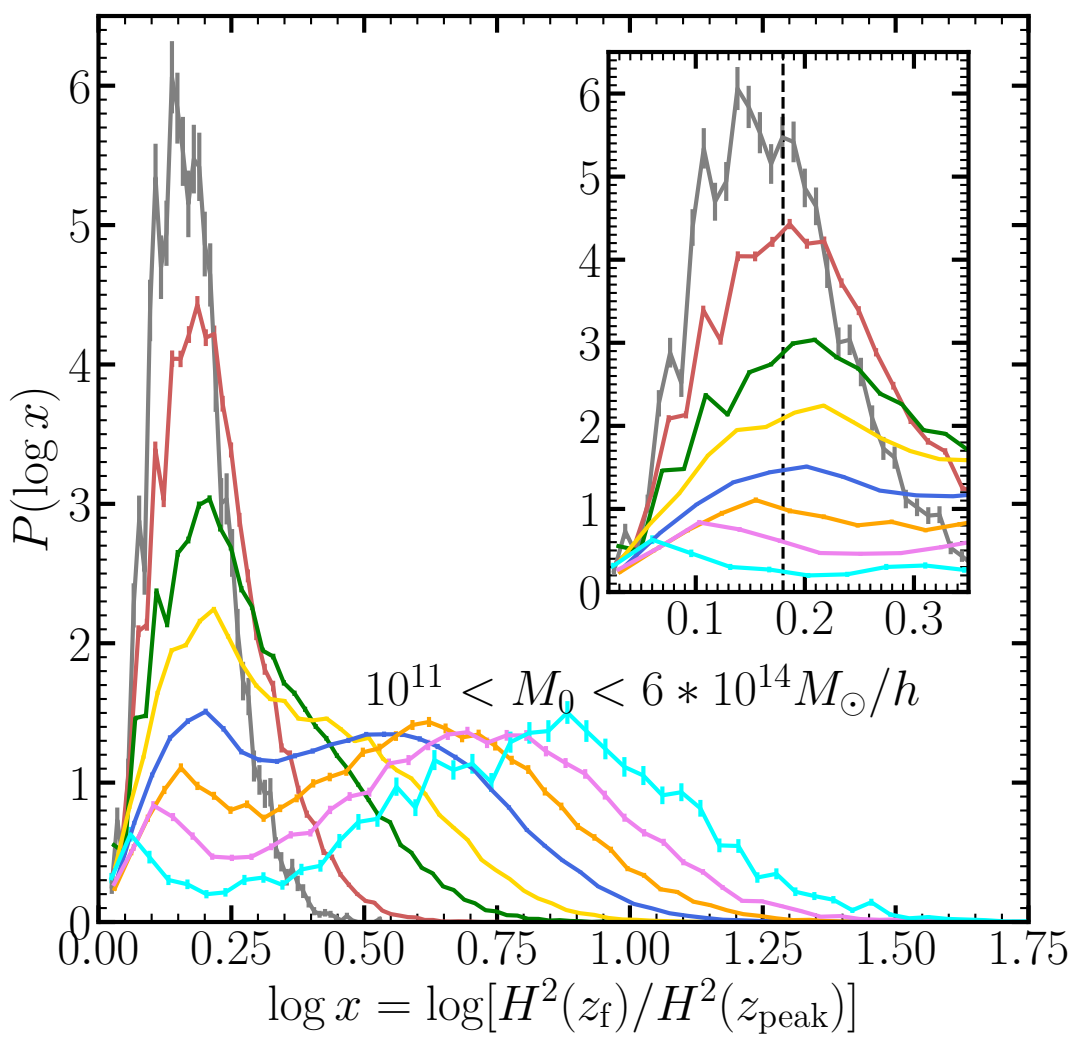

Figure 5. Similar to the left panel in Figure 2, except that now it shows the distribution of $\log x=\log \left[H^{2}\left(z_{\mathrm{f}}\right) / H^{2}\left(z_{\text {peak }}\right)\right]$ for various $z_{\text {peak }}$. The insert shows the $\log x$ distribution around the young population. The dashed vertical line indicates $\log x=0.18$. See text for the detail.

the $\log x$ distribution close to 1 . This indicates that only the old population halos at $z \sim 0$ satisfy the requirements that both $z_{\text {peak }}$ and $z_{\mathrm{f}}$ are much less than $z_{\mathrm{tp}}$. For the old population at high redshift, it is likely that their $z_{\mathrm{f}}$ are larger than or comparable to $z_{\mathrm{tp}}$. This means that these old halos in fact spend much or even most of their lifetimes in the fast accretion phase at $z>z_{\mathrm{f}}$. Finally, we note that the MAH shown in Eq. (3) is only a mean relation, and the scatter in it is quite large (see Zhao et al. 2003b) This may partly explain the dispersions of the distribution around the two peaks.

The above analysis suggests that the two populations identified in the $a_{\mathrm{nf}}$ distribution are directly related to the two phases of halo growth. It is thus interesting to see whether such bimodality also exists for the whole main halo population. Note again that the infall halo sample at $z_{\text {peak }}$ is a subset of the main halo population at the same redshift. We select main halos in two halo mass ranges $\left(10^{11}<M_{\text {vir }}<10^{11.5} h^{-1} \mathrm{M}_{\odot}\right.$ and $10^{12}<M_{\text {vir }}<10^{13} h^{-1} \mathrm{M}_{\odot}$ ) from four snapshots at $z \simeq 0.2,1,2,3$. We define the formation time $z_{\mathrm{f}}$ as the redshift, at which a halo reaches half of the mass at $z$ 
for the first time. Figure 6 shows the distribution of $a_{\mathrm{nf}}$ for these main halos, where $a_{\mathrm{nf}} \equiv\left(1+z_{\mathrm{f}}\right) /(1+z)$. For comparison, we also show in the same figure the results

for infall halos with $z_{\text {peak }} \simeq 0.2,1,2,3$ and with $M_{\text {peak }}$ in the same mass ranges. Since there is no information about the host halo mass for common main halos, we do not limit the host halo masses for infall halos in the comparison. In contrast to the infall halos, the $a_{\mathrm{nf}}$ distributions of the whole main halo populations are single-peaked for all the redshift and mass ranges considered. This is consistent with previous results that the formation time distribution is unimodal (e.g. Lacey \& Cole 1993; Lin et al. 2003).

In general, main halos tend to be older than the corresponding infall halos. At low redshift, the distributions for the main halos are similar to those of the old population of infall halos. Inspecting the distributions in detail, one can see a small bump at $a_{\mathrm{nf}} \sim 1.2$ for main halos. This suggests that the young population (in the fast accretion phase) does exist in the whole halo sample, though its fraction is much smaller than that in the infall halos. At high redshift $(z \geq 3)$, the distributions for main halos also peak at 1.2, similarly to those of infall halos. This is because most of the halos at high redshift tend to be in the fast accretion phase (see e.g. Zhao et al. 2003a). Moreover, we can find that the difference in the distribution of $a_{\mathrm{nf}}$ between the infall and main halos reaches its maximum at redshift between 1 and 2. This comparison clearly indicates that the presence of the bimodality in the formation time distribution is a property of infall halos that will eventually merge into a bigger halo. Apparently, the environment determined by the bigger halo can affect the formation histories of the smaller halos that will merge into it. We will come back to this question in a separate paper.

\section{NUMERICAL VS. SEMI-EMPIRICAL PREDICTIONS}

The above analysis is based on numerical simulations. Halo merger trees can also be constructed with the extended Press-Schechter formalism (Bond et al. 1991), and such a formalism has been widely used in studying halo MAHs and in semi-analytic models of galaxy formation (Kauffmann \& White 1993; Sheth \& Lemson 1999; Cole et al. 2000; Parkinson et al. 2008; Jiang \& van den Bosch 2016; Somerville \& Davé 2015). It is, therefore, also interesting to analyze if EPS merger trees have similar properties as the merger trees obtained from simulations. To this end, we use the code developed by Parkinson et al. (2008) ${ }^{1}$ to generate merger trees. Jiang \& van den Bosch (2014) compared several EPS merger tree generating codes, and found that the algorithm developed by Parkinson et al. (2008) agrees well with simulations in progenitor mass function, MAH, merger rate per descendant halo, and the un-evolved subhalo mass function. Here we test its performance in describing the MAHs of infall halos.

${ }^{1}$ http://star-www.dur.ac.uk/ cole/merger_trees/ 


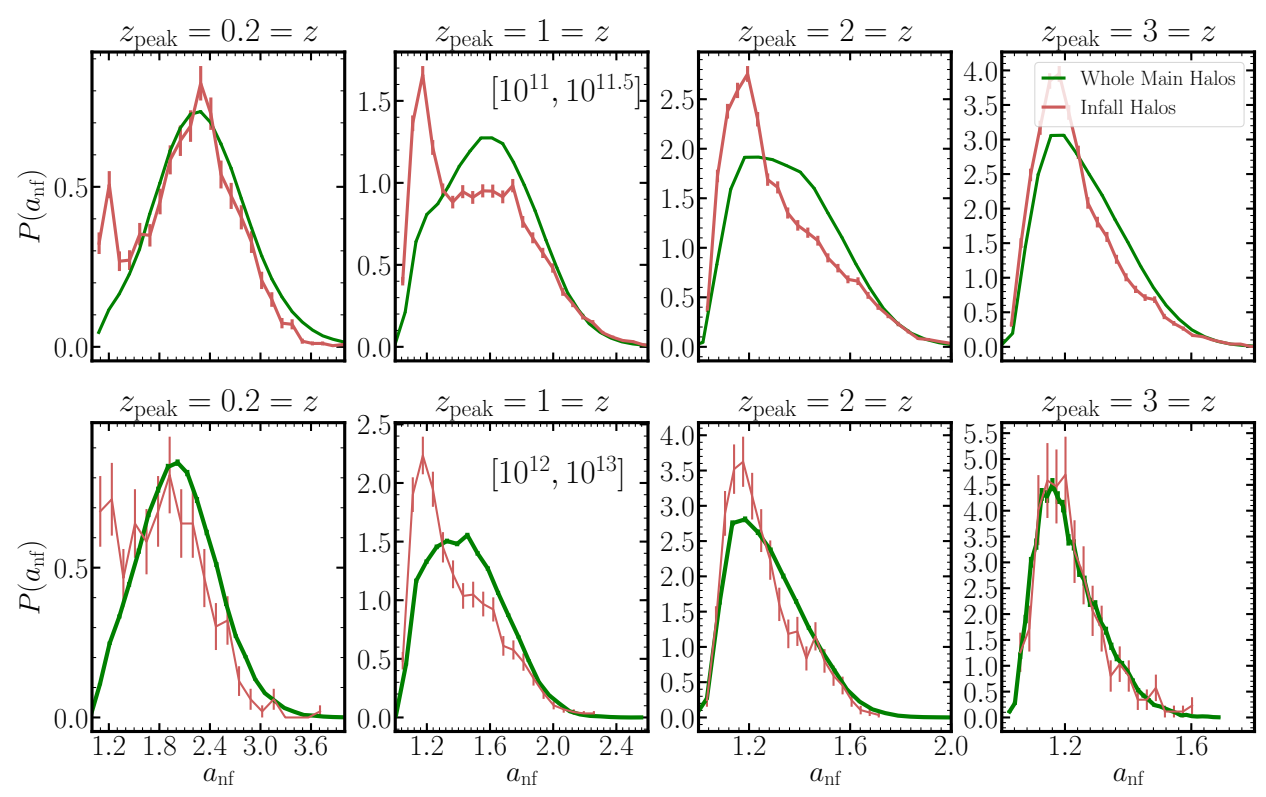

Figure 6. Formation time distributions for the whole main halos (green lines) and infall (red lines) halos at four redshifts as indicated in each panel. Note that for infall halos, $a_{\mathrm{nf}} \equiv\left(1+z_{\mathrm{f}}\right) /\left(1+z_{\text {peak }}\right)$, while for the whole main halos, $a_{\mathrm{nf}} \equiv\left(1+z_{\mathrm{f}}\right) /(1+z)$, where $z_{\text {peak }}$ (or $z$ ) is the redshift at which the infall halos (the main halos) are chosen. The upper panels show the results for small halos with halo masses in the range $\left[10^{11}, 10^{11.5}\right] h^{-1} \mathrm{M}_{\odot}$; the lower panels for larger halos with masses in $\left[10^{12}, 10^{13}\right] h^{-1} \mathrm{M}_{\odot}$. See text for more detail. Error bars show Poisson errors.

We generate 2000, 2000, 1000, and 100 merger trees for four host masses, $10^{11.25} h^{-1} \mathrm{M}_{\odot}, 10^{12.25} h^{-1} \mathrm{M}_{\odot}, 10^{13.25} h^{-1} \mathrm{M}_{\odot}$, and $10^{14.25} h^{-1} \mathrm{M}_{\odot}$, respectively. Consider a halo at the present time. The tree-generating program draws a set of random progenitor halos according to the progenitor halo mass function at a slightly earlier time. The procedure is repeated for each of the progenitors as we move back in time until the halo mass resolution limits, $7.29 \times 10^{5} h^{-1} \mathrm{M}_{\odot}, 7.29 \times 10^{6} h^{-1} \mathrm{M}_{\odot}$, $7.29 \times 10^{7} h^{-1} \mathrm{M}_{\odot}$, and $7.29 \times 10^{7} h^{-1} \mathrm{M}_{\odot}$ are reached correspondingly. The collection of all the progenitors at different times and their links form the merger tree of the halo. Any halo on the merger tree has only one descendant in the next snapshot but may have several progenitors at an earlier snapshot. The sum of the masses of all the progenitors is equal to the halo mass, and the most massive progenitor is called the main progenitor of the halo. Similarly, an infall halo is one on a sub-branch, and the accretion time is defined as the time when the infall halo is not the main progenitor of its descendant. Since the mass of a halo always grows with time in an EPS merger tree, the accretion time of an infall halo is exactly the same as the time when the infall halo reaches its maximum mass before accretion. The formation time is then defined as the time when the infall halo first reaches half of its maximum mass. Different from the merger trees in numerical simulations, the EPS merger trees are unable to 

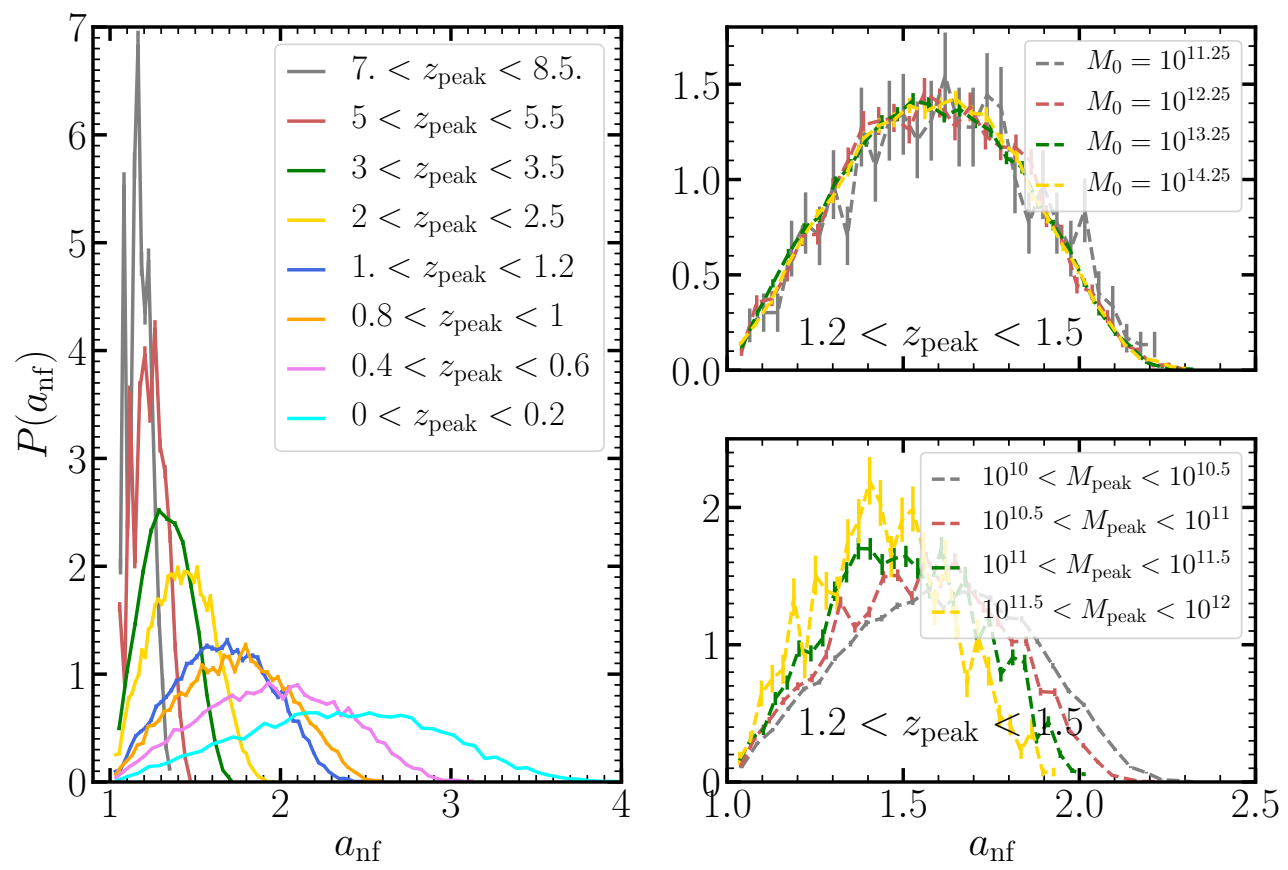

Figure 7. The same as Fig. 2, but for merger trees generated with EPS formalism. Error bars show Poisson errors.

trace the evolution of subhalos. In this case, there are no backsplash halos to exclude and we are not able to investigate whether or not an infall halo has crossed the virial radius of its descendant more than once.

Figure 7 shows the distributions of $a_{\mathrm{nf}}$ in similar $z_{\text {peak }}, M_{0}$, and $M_{\text {peak }}$ bins as shown in Fig. 2. A similar $M_{\text {peak }}$ threshold, i.e. $M_{\text {peak }}>100 m_{\mathrm{p}}=7.29 \times 10^{9} h^{-1} \mathrm{M}_{\odot}$, has been applied to the infall halo sample as well. The general trends with $z_{\text {peak }}, M_{\text {peak }}$ and $M_{0}$ are similar to those obtained from our simulation, but the bimodality is completely absent in the EPS merger trees. However, because of the differences in the definition of halos and in the treatment of halo accretion between the simulation and the EPS formalism, the exact cause of the discrepancy is unclear. One possibility is that the bimodality is the result of some environmental effects that are present in the simulation but not taken into account by the EPS formalism.

\section{SUMMARY AND DISCUSSION}

We investigate the assembly history of infall halos using high-resolution N-body simulations. These are halos on the branches of the halo merger trees before they are accreted into larger halos. We define the accretion redshift $\left(z_{\text {peak }}\right)$ of an infall halo as the redshift at which its halo mass reaches the peak value, and the formation redshift $\left(z_{\mathrm{f}}\right)$ as the redshift at which the infall halo reaches half of its peak mass for the first time. To compare the infall halos at different accretion time, we define a quantity $a_{\mathrm{nf}} \equiv\left(1+z_{\mathrm{f}}\right) /\left(1+z_{\text {peak }}\right)$ and examine its distribution. We find that, at a given 
accretion time, infall halos have bimodal distributions in $a_{\mathrm{nf}}$ and $z_{\mathrm{f}}$. The following is a list of our main results:

- According to the $a_{\mathrm{nf}}$ distribution, infall halos contain two distinct populations. For the first population (young population), the $a_{\text {nf }}$ distribution changes only slightly with $z_{\text {peak }}$, peaking at $a_{\text {nf }} \sim 1.2$. For the second population (old population), the peak value and the width both increase with decreasing $z_{\text {peak }}$, and are both larger than those of the young population.

- The infall halos are dominated by the young population at high redshift, while the old population becomes more and more important as the redshift decreases. At $z_{\text {peak }} \sim 2$, the two populations become comparable in number.

- Our analysis shows that the bimodal distribution naturally arises from the twophase accretion histories of dark matter halos. The young population consists of halos that are still in the fast accretion phase at the time of accretion, while halos in the old population have already entered slow accretion phase at the time of accretion.

- We have also studied the assembly histories of common individual halos without distinguishing whether they will be accreted or not. No significant bimodal feature is found in the distribution of their formation redshifts. This indicates that the environments defined by the host halo may affect the formation histories of its subhalos even before they are accreted into the host.

- We have also checked the merger trees generated with the EPS formalism and found that the infall halos in such merger trees do not show bimodal distribution in formation redshifts. This difference between EPS and $N$-body merger trees may be caused by the fact that environmental effects, which are taken into account in the simulation but not in the EPS formalism, are important in the formation and evolution of infall halos.

It is well known that galaxies exhibit bimodal distributions in their colors and star formation rates (SFR; e.g. Strateva et al. 2001; Blanton et al. 2003; Baldry et al. 2004; Brinchmann et al. 2004). In the current scenario of galaxy formation, galaxy properties are expected to correlate with the assembly histories of their host halos. For example, the age-matching model assumes that older halos tend to host galaxies with older stellar populations (Hearin \& Watson 2013; Hearin et al. 2014; Watson et al. 2015). This simple model successfully reproduces the trends of galaxy color with a variety of galaxy statistics, such as galaxy clustering and the galaxygalaxy lensing signal. Hydrodynamical simulations (Bray et al. 2016) also reveal a correlation between the assembly histories of galaxies and those of their halos. In addition, halo spins and concentrations are strongly correlated with halo formation time (Zhao et al. 2003b; Wechsler et al. 2002; Wang et al. 2011; Hahn et al. 2007). These two halo parameters are thought to play an important role in shaping the disc 
size and the surface density of gas in galaxy disks (Mo et al. 1998; Guo et al. 2011; Croton et al. 2016; Henriques et al. 2015), and thus potentially having effects on galaxy morphology. The bimodal distribution in the formation time of infall halos we have found may, therefore, provide insight into the origin of these two distinct galaxy populations, particularly the origin of color bimodality found for satellite galaxies (van den Bosch et al. 2008). We emphasize, however, that only infall halos have clearly bimodal formation time distributions, and that there is considerable scatter between galaxy color/SFR and the formation time of the host halo (see e.g. Wang et al. 2017). Clearly, the relation between the bimodality in halo formation and that in galaxy color merits further study.

Once infall halos are accreted by larger halos, their central galaxies become satellites. These satellites are expected to undergo some satellite-specific quenching and morphology-transformation processes. These processes have been constrained by comparing the properties of satellites to those of central galaxies in control samples (van den Bosch et al. 2008; Weinmann et al. 2009; Pasquali et al. 2010; Wetzel et al. 2012; Peng et al. 2012; Knobel et al. 2013; Bluck et al. 2014; Fossati et al. 2017). Most of the control samples were made so that the centrals in the control sample had the same stellar mass and redshift distributions as the satellites; Wetzel et al. (2013) also accounted for evolution of the centrals. However, the assumption underlying all these studies is that, before they are accreted, satellites are the same as central galaxies of the same stellar mass. This assumption is not supported by our finding that on average, infall halos are younger than the whole halo population of the same mass. If galaxy properties such as star formation rate and color, indeed correlate with the assembly histories of their host halos, then previous studies will underestimate the efficiency of satellite-specific quenching processes. Clearly, well controlled samples of centrals and satellites, matched not only in stellar mass (and/or halo mass), but also in the mass assembly history, are needed (see Mistani et al. (2016)). Our results should, therefore, be useful in interpreting the observational data in terms of satellite quenching processes.

\section{ACKNOWLEDGEMENTS}

We thanks the anonymous referee for a useful report that significantly improve the presentation of this paper. J. Shi thanks J. Tinker for helpful discussion, and the hospitality of Nordita during the program of "Advances in Theoretical Cosmology in Light of Data". She also thanks UMass and UPenn for their hospitality in late 2016. RKS thanks the ICTP for its hospitality during summer 2017. The Phoenix and Aquarius data were provided by the Virgo Consortium. We thank the GALFORM team for making the EPS merger tree generating code publicly available. This work is supported by 973 Program (2015CB857002), NSFC $(11522324,11733004,11421303,11673015)$, and the Fundamental Research Funds for the Central Universities. H.J.M. acknowledges the support of NSF AST-1517528. 
The work is also supported by the Supercomputer Center of the University of Science and Technology of China.

\section{REFERENCES}

Abadi, M. G., Moore, B., \& Bower, R. G. 1999, MNRAS, 308, 947

Allgood, B., Flores, R. A., Primack, J. R., et al. 2006, MNRAS, 367, 1781

Baldry, I. K., Glazebrook, K., Brinkmann, J., et al. 2004, ApJ, 600, 681

Benson, A. J. 2005, MNRAS, 358, 551

Blanton, M. R., Eisenstein, D., Hogg, D. W., Schlegel, D. J., \& Brinkmann, J. 2005, ApJ, 629, 143

Blanton, M. R., Hogg, D. W., Bahcall, N. A., et al. 2003, ApJ, 594, 186

Bluck, A. F. L., Mendel, J. T., Ellison, S. L., et al. 2014, MNRAS, 441, 599

Bond, J. R., Cole, S., Efstathiou, G., \& Kaiser, N. 1991, ApJ, 379, 440

Boylan-Kolchin, M., Springel, V., White, S. D. M., Jenkins, A., \& Lemson, G. 2009, MNRAS, 398, 1150

Bray, A. D., Pillepich, A., Sales, L. V., et al. 2016, MNRAS, 455, 185

Brinchmann, J., Charlot, S., White, S. D. M., et al. 2004, MNRAS, 351, 1151

Chandrasekhar, S. 1943, ApJ, 97, 255

Chaves-Montero, J., Angulo, R. E., Schaye, J., et al. 2016, MNRAS, 460, 3100

Cole, S., Lacey, C. G., Baugh, C. M., \& Frenk, C. S. 2000, MNRAS, 319, 168

Conroy, C., Wechsler, R. H., \& Kravtsov, A. V. 2006, ApJ, 647, 201

Croton, D. J., Stevens, A. R. H., Tonini, C., et al. 2016, ApJS, 222, 22

Davis, M., Efstathiou, G., Frenk, C. S., \& White, S. D. M. 1985, ApJ, 292, 371

Fossati, M., Wilman, D. J., Mendel, J. T., et al. 2017, ApJ, 835, 153

Gan, J., Kang, X., van den Bosch, F. C., \& Hou, J. 2010, MNRAS, 408, 2201

Gao, L., Navarro, J. F., Frenk, C. S., et al. 2012, MNRAS, 425, 2169

Gao, L., Springel, V., \& White, S. D. M. 2005, MNRAS, 363, L66
Gao, L., \& White, S. D. M. 2007, MNRAS, 377, L5

Gao, L., White, S. D. M., Jenkins, A., Stoehr, F., \& Springel, V. 2004, MNRAS, 355, 819

Gill, S. P. D., Knebe, A., \& Gibson, B. K. 2005, MNRAS, 356, 1327

Giocoli, C., Tormen, G., \& van den Bosch, F. C. 2008, MNRAS, 386, 2135

Gunn, J. E., \& Gott, III, J. R. 1972, ApJ, 176,1

Guo, Q., White, S., Boylan-Kolchin, M., et al. 2011, MNRAS, 413, 101

Hahn, O., Porciani, C., Carollo, C. M., \& Dekel, A. 2007, MNRAS, 375, 489

Han, J., Cole, S., Frenk, C. S., \& Jing, Y. 2016, MNRAS, 457, 1208

Hashimoto, Y., Funato, Y., \& Makino, J. 2003, ApJ, 582, 196

Hayashi, E., Navarro, J. F., Taylor, J. E., Stadel, J., \& Quinn, T. 2003, ApJ, 584, 541

Hearin, A. P., Behroozi, P. S., \& van den Bosch, F. C. 2016, MNRAS, 461, 2135

Hearin, A. P., \& Watson, D. F. 2013, MNRAS, 435, 1313

Hearin, A. P., Watson, D. F., Becker, M. R., et al. 2014, MNRAS, 444, 729

Henriques, B. M. B., White, S. D. M., Thomas, P. A., et al. 2015, MNRAS, 451, 2663

Hinshaw, G., Larson, D., Komatsu, E., et al. 2013, ApJS, 208, 19

Jiang, C. Y., Jing, Y. P., Faltenbacher, A., Lin, W. P., \& Li, C. 2008, ApJ, 675, 1095

Jiang, F., \& van den Bosch, F. C. 2014, MNRAS, 440, 193

—. 2016, MNRAS, 458, 2848

Jiang, L., Cole, S., Sawala, T., \& Frenk, C. S. 2015, MNRAS, 448, 1674

Jing, Y. P., \& Suto, Y. 2002, ApJ, 574, 538

Jing, Y. P., Suto, Y., \& Mo, H. J. 2007, ApJ, 657, 664 
Kauffmann, G., \& White, S. D. M. 1993, MNRAS, 261, doi:10.1093/mnras/261.4.921

Knobel, C., Lilly, S. J., Kovač, K., et al. 2013, ApJ, 769, 24

Lacey, C., \& Cole, S. 1993, MNRAS, 262, 627

Li, R., Gao, L., Xie, L., \& Guo, Q. 2013, MNRAS, 435, 3592

Li, S.-J., Zhang, Y.-C., Yang, X.-H., et al. 2016, Research in Astronomy and Astrophysics, 16, 130

Li, Y., \& Mo, H. 2009, ArXiv e-prints, arXiv:0908.0301

Li, Y., Mo, H. J., \& Gao, L. 2008, MNRAS, 389, 1419

Lin, W. P., Jing, Y. P., \& Lin, L. 2003 , MNRAS, 344, 1327

Ludlow, A. D., Navarro, J. F., Springel, V., et al. 2009, ApJ, 692, 931

Mistani, P. A., Sales, L. V., Pillepich, A., et al. 2016, MNRAS, 455, 2323

Mo, H., van den Bosch, F. C., \& White, S. 2010, Galaxy Formation and Evolution

Mo, H. J., Mao, S., \& White, S. D. M. 1998, MNRAS, 295, 319

Nagai, D., \& Kravtsov, A. V. 2005, ApJ, 618,557

Oguri, M., \& Lee, J. 2004, MNRAS, 355, 120

Paranjape, A., Kovač, K., Hartley, W. G., \& Pahwa, I. 2015, MNRAS, 454, 3030

Parkinson, H., Cole, S., \& Helly, J. 2008, MNRAS, 383, 557

Pasquali, A., Gallazzi, A., Fontanot, F., et al. 2010, MNRAS, 407, 937

Peng, Y.-j., Lilly, S. J., Renzini, A., \& Carollo, M. 2012, ApJ, 757, 4

Quilis, V., Moore, B., \& Bower, R. 2000, Science, 288, 1617

Sheth, R. K. 2003, MNRAS, 345, 1200

Sheth, R. K., \& Lemson, G. 1999, MNRAS, 305, 946

Sheth, R. K., \& Tormen, G. 2004, MNRAS, 350, 1385

Shi, J., Wang, H., \& Mo, H. J. 2015, ApJ, 807,37

Somerville, R. S., \& Davé, R. 2015, ARA\&A, 53, 51

Springel, V. 2005, MNRAS, 364, 1105
Springel, V., White, S. D. M., Tormen, G., \& Kauffmann, G. 2001, MNRAS, 328, 726

Springel, V., White, S. D. M., Jenkins, A., et al. 2005, Nature, 435, 629

Springel, V., Wang, J., Vogelsberger, M., et al. 2008, MNRAS, 391, 1685

Strateva, I., Ivezić, Ž., Knapp, G. R., et al. 2001, AJ, 122, 1861

Taylor, J. E., \& Babul, A. 2004, MNRAS, 348,811

Tinker, J., Kravtsov, A. V., Klypin, A., et al. 2008, ApJ, 688, 709

Tormen, G. 1997, MNRAS, 290, 411

van den Bosch, F. C. 2017, MNRAS, 468, 885

van den Bosch, F. C., Aquino, D., Yang, X., et al. 2008, MNRAS, 387, 79

Vitvitska, M., Klypin, A. A., Kravtsov, A. V., et al. 2002, ApJ, 581, 799

Vogelsberger, M., Genel, S., Springel, V., et al. 2014, MNRAS, 444, 1518

Wang, H., Mo, H. J., \& Jing, Y. P. 2009, MNRAS, 396, 2249

Wang, H., Mo, H. J., Jing, Y. P., Yang, X., \& Wang, Y. 2011, MNRAS, 413, 1973

Wang, H., Mo, H. J., Chen, S., et al. 2017, ArXiv e-prints, arXiv:1707.09002

Wang, H. Y., Jing, Y. P., Mao, S., \& Kang, X. 2005, MNRAS, 364, 424

Wang, H. Y., Mo, H. J., \& Jing, Y. P. 2007, MNRAS, 375, 633

Watson, D. F., Hearin, A. P., Berlind, A. A., et al. 2015, MNRAS, 446, 651

Wechsler, R. H., Bullock, J. S., Primack, J. R., Kravtsov, A. V., \& Dekel, A. 2002, ApJ, 568, 52

Weinmann, S. M., Kauffmann, G., van den Bosch, F. C., et al. 2009, MNRAS, 394, 1213

Wetzel, A. R. 2011, MNRAS, 412, 49

Wetzel, A. R., Tinker, J. L., \& Conroy, C. 2012, MNRAS, 424, 232

Wetzel, A. R., Tinker, J. L., Conroy, C., $\&$ van den Bosch, F. C. 2013, MNRAS, 432,336

Wyder, T. K., Martin, D. C., Schiminovich, D., et al. 2007, ApJS, 173, 293 
Xie, L., \& Gao, L. 2015, MNRAS, 454, 1697

Zhao, D. H., Jing, Y. P., Mo, H. J., \& Börner, G. 2003a, ApJL, 597, L9
Zhao, D. H., Mo, H. J., Jing, Y. P., \& Börner, G. 2003b, MNRAS, 339, 12 